\title{
Nodular Lesions on Legs: A Dermoscopic Surprise
}

\section{${ }^{1}$ Balachandra S Ankad, ${ }^{2}$ Savitha L Beergouder}

How to cite this article: Ankad BS, Beergouder SL. Nodular Lesions on Legs: A Dermoscopic Surprise. Int J Dermoscop 2017;1(2):60-61.

\section{Source of support: Nil}

\section{Conflict of interest: None}

\section{Sir,}

Dermoscopy helps to see structures and features invisible to the naked eye, providing additional morphologic information during clinical examination of skin lesion. It is rightly called as dermatologist stethoscope. It facilitates the clinical recognition of several inflammatory and infectious diseases, as well as their discrimination from skin tumors. ${ }^{1}$ Sometimes it can surprise with unusual yet interesting findings. Here authors report a dermoscopic surprise wherein etiology of itchy lesion was identified.

A 21-year-old male presented to dermatology outpatient department with intensely pruritic lesions over both the legs for the past 1 month. Lesions initially started as itchy papules that slowly progressed into nodulation. Itching was severe disturbing his daily routine. There was no history of similar complaints in the family and no history of systemic disease, immunodeficiency, or drug intake. On examination, multiple excoriated hyperpigmented papules and nodules measuring $0.5 \times 1 \mathrm{~cm}$ with symmetrical distribution over both lower limbs were noted (Figs 1A and 2A). Palms and soles and mucosal examination were normal. Systemic examination revealed no significant abnormality. Dermoscopy of the lesions by handheld dermoscope (with 10× magnification) revealed "white star burst" pattern, white scales, and hemorrhagic spots. Brownish striations were noted at the periphery (Figs 1B and 2B). To our surprise, lice were observed at the periphery of the lesion. They were multiple and scattered around the lesion. Dermoscopy could demonstrate only some parts of lice as they were embedded in the

\footnotetext{
${ }^{1}$ Professor and Head, ${ }^{2}$ Senior Resident

1,2Department of Dermatology, S. Nijalingappa Medical College and H.S.K Hospital \& Research Centre, Bagalkot, Karnataka India
}

Corresponding Author: Balachandra S Ankad, Professor and Head, Department of Dermatology, S. Nijalingappa Medical College and H.S.K Hospital \& Research Centre, Bagalkot Karnataka, India, Phone: +919980410056, e-mail: drbsankad@ gmail.com epidermis. Skin biopsy was done and histopathology showed features consistent with prurigo nodularis (PN) (Fig. 3). Patient was advised about personal hygiene, he was asked to discard the infested clothes or wash them in hot water and dry them in bright sunlight, followed by topical potent steroids and antihistamines for PN lesions.

Prurigo nodularis is a chronic neurodermatitis that presents with intensely pruritic nodules secondary to an intense itch-scratch cycle. ${ }^{2}$ The pathogenesis of PN is ambiguous, although immunoneuronal cross-talk is implicated. Its etiology was found to be heterogeneous. It can emerge as the symptom of various dermatological, neurological, psychiatric, and systemic diseases. ${ }^{3}$ Dermoscopy of early excoriated lesions of PN shows pearly white areas in the center extending peripherally in a "star burst pattern," white scale, erosion, crust, dotted
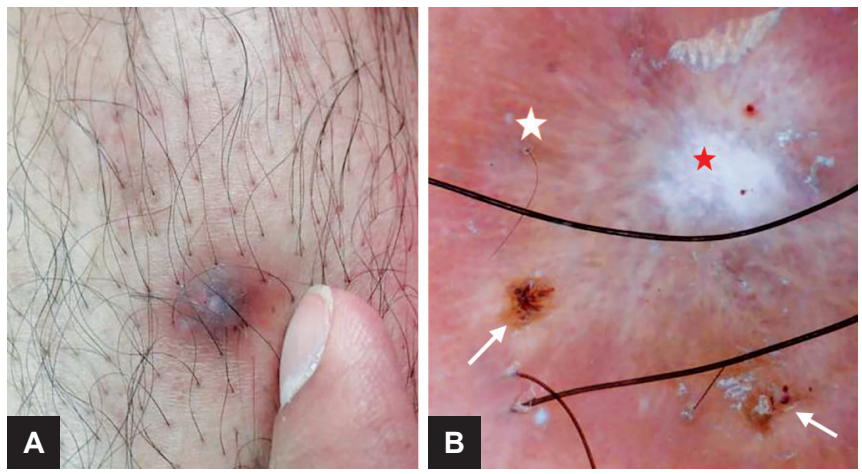

Figs 1A and B: (A) Clinical image of PN; (B) Dermoscopy shows "star burst" pattern of white areas (red star), parts of lice (white arrows), brownish striations (white star), and white scales
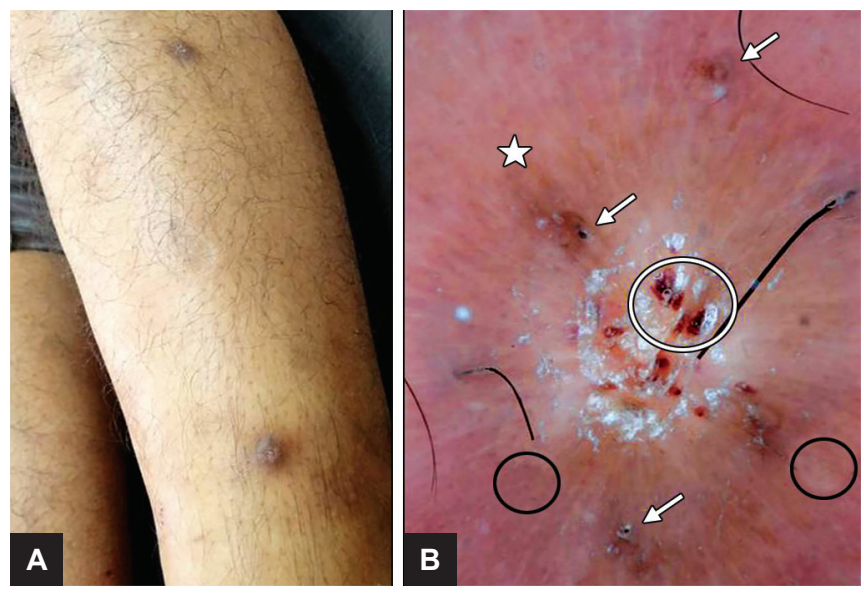

Figs 2A and B: (A) Hyperkeratotic nodules of $\mathrm{PN}$; (B) dermoscopy shows red globules (white circle), parts of lice (white arrows), red dots (black circles), brownish striations (white star), and white scales 


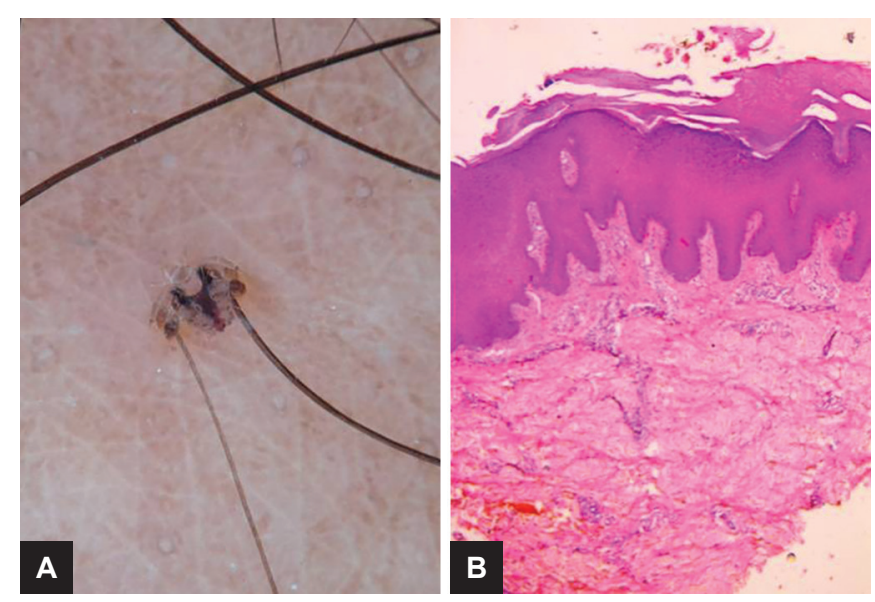

Figs $3 A$ and B: Dermoscopic image of pubic louse snugly attached to the hairs: (A) Histopathology of PN showing hyperkeratosis, acanthosis, epidermal hyperplasia, and collagen bundles (B) hematoxylin and eosin 10x

or glomerular vessels, and hemorrhagic spots. Late and hyperkeratotic lesions demonstrate similar patterns but with pronounced white areas. ${ }^{2,4}$ In this case, typical dermoscopic patterns of PN were observed and lice were found accidently at the periphery of all the lesions of PN. Mouth, forelegs, and proximal abdominal parts of lice were noted. However, it was not sure whether they were pubic or body lice as only parts of lice were visible. Though lice infestation can be diagnosed by naked eye examination or using magnifying lens, the patient presented purely with nodular lesions confined to lower legs. Only after dermoscopic examination, we could observe the lice. As cause for intense pruritus was not recognized, we strongly believe that pruritic nodular lesions were the result of infestation by lice in this patient. Hence, dermoscopy helped us to identify one of the treatable causes of intense itching.

To conclude, dermoscopic findings should always be interpreted in the clinical context of the patient, integrated with information from the history and macroscopic and microscopic examination. It definitely improves the clinical recognition of several inflammatory and infectious dermatoses, thus becoming an irreplaceable clinical tool for dermatologists.

\section{REFERENCES}

1. Lallas A, Giacomel J, Argenziano G. Dermoscopy in general dermatology: practical tips for the clinician. Br J Dermatol 2014 Mar;170(3):514-526.

2. Ankad BS, Beergouder SL. Hypertrophic lichen planus versus prurigo nodularis: a dermoscopic perspective. Dermatol Pract Concept 2016 Apr;6(2):3.

3. Lee MR, Shumack S. Prurigo nodularis: a review. Australas J Dermatol 2005 Nov;46(4):211-218.

4. Errichetti E, Piccirillo A, Stinco G. Dermoscopy of prurigo nodularis. J Dermatol 2015 Jun;42(6):632-634. 